\title{
A Mathematical Approach for Hidden Node Problem in Cognitive Radio Networks
}

\author{
Felix Obite ${ }^{1}$, Kamaludin Mohammad Yusof ${ }^{2}$, Jafri Din $^{\star 3}$ \\ ${ }^{1,2}$ Department of Communication, Faculty of Electrical Engineering, Universiti Teknologi Malaysia \\ ${ }^{3}$ Wireless Communication Center, Faculty of Electrical Engineering, Universiti Teknologi Malaysia \\ ${ }^{1}$ Department of Physics, Faculty of Science, Ahmadu Bello University, Zaria, Nigeria \\ ${ }^{*}$ Corresponding author, e-mail: jafri@utm.my
}

\begin{abstract}
Cognitive radio (CR) technology has emerged as a realistic solution to the spectrum scarcity problem in present day wireless networks. A major challenge in CR radio networks is the hidden node problem, which is the inability of the CR nodes to detect the primary user. This paper proposes energy detector-based distributed sequential cooperative spectrum sensing over Nakagami-m fading, as a tool to solve the hidden node problem. The derivation of energy detection performance over Nakagami-m fading channel is presented. Since the observation represents a random variable, likelihood ratio test (LRT) is known to be optimal in this type of detection problem. The LRT is implemented using the Neyman-Pearson Criterion (maximizing the probability of detection but at a constraint of false alarm probability). The performance of the proposed method has been evaluated both by numerical analysis and simulations. The effect of cooperation among a group of $C R$ nodes and system parameters such as SNR, detection threshold and number of samples per $C R$ nodes is investigated. Results show improved detection performance by implementing the proposed model.
\end{abstract}

Keywords: Mathematical Approach, CR, Hidden Node Problem, Nakagami-m Fading, Neyman-Pearson Criterion

Copyright @ 2017 Universitas Ahmad Dahlan. All rights reserved.

\section{Introduction}

Over the last decades, the radio spectrum has become increasingly crowded,

particularly as new wireless applications and technologies are continuously being developed [1]. The present spectrum allocation strategy worked perfectly in the past since it provided an optimum solution by reducing interference between active wireless users. As a result, it became eminent to address the efficiency of using the scarce radio spectrum which was discussed intensely by several radio regulatory authorities around the world. The existing spectrum policy has full allocation but poor utilization. According to this policy, spectrum bands are assigned to some licensed users on a long term basis. In most cases, the assigned spectrum bands are reserved over vast geographical regions. Hence, unlicensed spectrum is becoming more scarce and insufficient to accommodate the increasing spectrum demand. Interestingly, studies have revealed that a large portion of the radio spectrum is underutilized at different periods of time under different geographic locations [2]. This resulted to spectrum white spaces or spectrum holes. Such unused radio spectrum is used by secondary users when the primary users are not utilizing the spectrum. This concept gave rise to dynamic spectrum access (DSA) [3]. A given $\mathrm{CR}$ radio can access the radio spectrum opportunistically on a demand basis whenever a given portion of the radio spectrum is free, hence increasing the spectrum utilization.

The key technology enabling unlicensed users to access the licensed spectrum in a dynamic and opportunistic pattern, without interfering with the existing users is the cognitive radio (CR) technology. In order for secondary users (unlicensed user) to intelligently and dynamically employ such unused spectrum without interference with the primary users (licensed users), the secondary radio must be able to learn in real-time the occupancy of the spectrum. Through learning, the radios are able to think and adapt intelligently [4]. To this end, the secondary users require an efficient and robust spectrum sensing to identify unused frequency bands and the ability to detect primary users as soon as they become noticeable in the frequency band [5]. 
Due to shadowing and deep fading effects in wireless communications, or when the cognitive radio nodes are located further away from the primary user, the signal power received by the cognitive radio node from the primary user terminals can become very low such that the primary user is unable to be detected. The inability of the CR nodes to detect the PU in such a scenario is known as the hidden node problem in CR.

The rest of the paper is organized as follows. In Section 2, the motivation regarding this research and review of the current related literature are presented. Section 3 discusses the system model, the basic principle and criteria used in statistical decision theory are presented. Simulation and results are covered in Section 4, and finally, we conclude the paper in Section 5.

\section{Motivation and Related Work}

The increasing demand for additional bandwidth to support existing and new services has led to the current spectrum scarcity and spectrum underutilization. Three main paths shape the research study embarked by wireless communication practitioners over the last decades to arrive at an effective spectrum sensing mechanism, namely transmitter detection or noncooperative techniques, cooperative detection techniques and interference based detection techniques. The transmitter detection techniques comprise of energy detection, cyclostationary detection and matched filter detection [6]. These methods are classified further as coherent and non-coherent, meaning that they have either complete, partial or no a priori knowledge of the primary transmitter respectively [7]. Cooperative schemes consist of centralized, distributed and relay assisted or cluster based detection techniques. While transmitter and cooperative detection techniques "sniff" the spectrum to minimize primary transmitter interference; interference based detection aim at minimizing interference to the primary receiver. In noncooperative sensing [8], each secondary user (SU) depends solely on its ability to detect the presence of a primary user (PU) transmission on a specific spectrum band. Energy detection [9], [10, 11] was proposed in literature as a method of detecting the presence of a PU transmission without cooperation between the SUs. In energy detection, prior knowledge about the PU is not required to determine if the channel is occupied or not. It has the advantage of low computational complexity and easy to implement, $[12,13]$. However, the detection performance suffers from two major drawbacks, 1) the sensing node inability to detect the noise variance. Thus, the SU may experience false-alarm particularly in noisy environments. 2) Silence synchronization: the sensing node inability to distinguish between a PU transmission and another SU transmission.

In a cognitive radio system, the authors in [14] established that cooperative sensing improves the reliability of detecting a PU and also capable of combating the hidden node problem in deep fading and shadowing channel. The article in [3] resolved that there is always a compromise between detection performance and shadowing, receiver uncertainty problem and multipath fading. Therefore to combat this problem, by exploiting spatial diversity, cooperative spectrum sensing will improve detection performance significantly.

Distributed cooperative spectrum sensing is a well-researched area in the literature of wireless sensor networks (WSNs) [1], [15-19]. We can adopt such detection strategy in the context of CR networks. The authors in [20] discuss wideband detection by focusing on multiple frequency bands using distributed methods. Noisy reporting channels between each CR node for distributed sensing strategy is addressed in [21]. Energy-efficient distributed sensing and access scheme are presented in [22], while distributed spectrum sensing security issues are addressed in [23]. An implementation model and procedure using SDR for actualizing distributed sensing are presented in [24].

The Nakagami-m distribution model [25], gives the best fit to indoor mobile and mobile multipath propagation [26-29]. While energy detection distributed spectrum sensing have been studied extensively in the last years, performance using the proposed mathematical approach is relatively less investigated. Thus, this paper proposes distributed sequential cooperative spectrum sensing over Nakagami-m fading for solving the hidden node problem in cognitive radio networks.

TELKOMNIKA Vol. 15, No. 3, September 2017: $1127-1136$ 


\section{System Model}

This section presents the system model for energy detector-based distributed sequential cooperative sensing over Nakagami-m fading channel. The basic principle and criteria used in statistical decision theory are investigated. The derivation of energy detection performance over Nakagami-m fading channel is revisited.

With distributed sequential sensing model [30-33], the CR senses the spectrum and sends the information to the next $\mathrm{CR}$ in a sequential pattern. As shown in Figure 1, the $(n-1)^{\text {th }}$ $\mathrm{CR}$ node senses the spectrum and sends the information to the adjacent $(n)^{\text {th }}$ CR node, which then fuses the $d_{n-1}$ received information with its own $y_{n}$ local sensing information and sends it to the next $(n+1)^{\text {th }} \mathrm{CR}$ node. Using such structure, the last node in the sequence ( $N^{\text {th }}$ node) gives a final binary decision on the primary user presence.

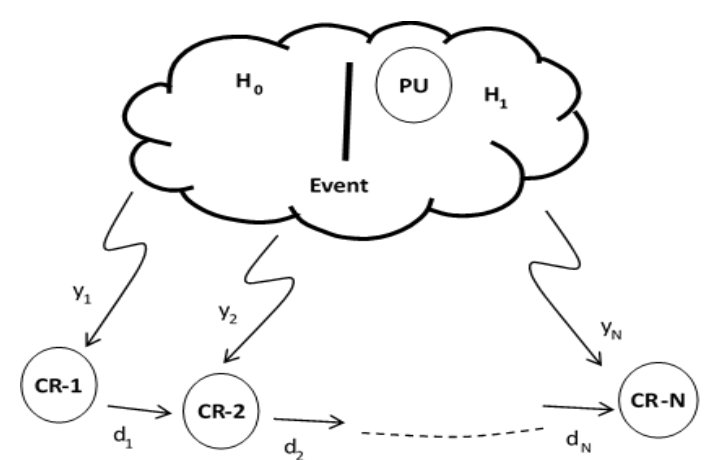

Figure 1. Distributed sequential spectrum sensing

Using the likelihood function, the detection criteria is formulated and presented subsequently.

The $(n-1)^{\text {th }}$ node gives a binary decision using its own sensing and received information from its previous node denoted by $d_{n-1}=1$ if its decision is $H_{1}$ and $d_{n-1}=0$ if its decision is $H_{0}$. Suppose $y_{n}$ and $d_{n-1}$ joint distributions under hypotheses $H_{0}$ and $H_{1}$ are given by the expression $f_{y_{n}, d_{n-\| H_{0}}}\left(y_{n}, d_{n-1} \mid H_{0}\right)$ and $f_{y_{n}, d_{n-\| H_{1}}}\left(y_{n}, d_{n-1} \mid H_{1}\right)$ respectively, hence the likelihood ratio may be expressed as [30]:

$$
\Lambda\left(y_{n}, d_{n-1}\right)=\frac{f_{y_{n}, d_{n-1 \mid H_{1}}}\left(y_{n}, d_{n-1} \mid H_{1}\right)}{f_{y_{n}, d_{n-\| H}}\left(y_{n}, d_{n-1} \mid H_{\mathrm{o}}\right)}
$$

The expression can be remodified by assuming conditional independence between $y_{n}$ and $d_{n-1}$ as [30]:

$$
\Lambda\left(y_{n}, d_{n-1}\right)=\frac{f_{y_{n}, d_{n-l \mid H_{1}}}\left(y_{n}, d_{n-1} \mid H_{1}\right)\left[P_{d, n-1} \delta\left(d_{n-1}-1\right)+\left(1-P_{d, n-1}\right) \delta\left(d_{n-1}\right)\right]}{f_{y_{n}, d_{n-1 H_{0}}}\left(y_{n}, d_{n-1} \mid H_{0}\right)\left[P_{f a, n-1} \delta\left(d_{n-1}-1\right)+\left(1-P_{f a, n-1}\right) \delta\left(d_{n-1}\right)\right]}
$$

Where the probability of detection is $P_{d, n-1}$ and the probability of false alarm is $P_{f a, n-1}$ at the $(n-1)^{\text {th }}$ CR node respectively, which is given by:

$$
P_{d, n-1}=P_{r}\left\{d_{n-1}=1 \mid H_{1}\right\}
$$




$$
P_{f a, n-1}=P_{r}\left\{d_{n-1}=1 \mid H_{0}\right\}
$$

And $\delta(x)$ denotes the dirac delta function expressed as $\delta(x)=1$ for $x=0$ and $\delta(x)=0$ for $x=1$. Thus, based on $d_{n-1}$ received information, the $n^{\text {th }}$ CR node may have two detection criteria expressed as:

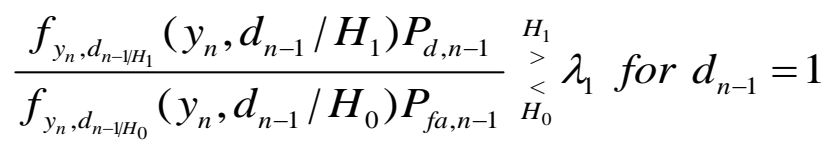

$$
\begin{aligned}
& \frac{f_{y_{n}, d_{n-\| H_{1}}}\left(y_{n}, d_{n-1} \mid H_{1}\right)\left(1-P_{d, n-1}\right)}{f_{y_{n}, d_{n-\| H_{0}}}\left(y_{n}, d_{n-1} \mid H_{0}\right)\left(1-P_{f a, n-1}\right)} \underset{H_{0}}{\stackrel{H_{1}}{<}} \lambda_{2} \text { for } d_{n-1}=0
\end{aligned}
$$

Based on the distribution models considered in the system, $\lambda_{1}$ and $\lambda_{2}$ are detection thresholds that can be determined.

\subsection{Performance Metrics}

Based on the binary hypothesis, the performance of energy detector is categorized by the following metrics.

1. Probability of detection $\left(P_{d}\right)$ : probability of deciding that the signal is present when $H_{1}$ is true, given by [30]:

$$
P_{d}=\left[\Lambda>\lambda \mid H_{1}\right]
$$

2. Probability of false alarm $\left(P_{f a}\right)$ : probability of deciding that the signal is present while in actual sense $H_{0}$ is true, given by:

$$
P_{f a}=\operatorname{Pr}\left[\Lambda>\lambda \mid H_{0}\right]
$$

In cognitive radio context, a false alarm indicates undetected spectrum holes. Thus a high $P_{f a}$ value signifies low spectrum usage by SUs.

1. Probability of missed detection $\left(P_{m d}\right)$ : probability of deciding that the signal is absent when $H_{1}$ is true, given by:

$$
P_{m d}=\operatorname{Pr}\left[\Lambda<\lambda \mid H_{1}\right]
$$

Which is the same as detecting a spectrum hole when there is none. By consequence, a high $P_{m d}$ value implies unexpected interference to PUs.

\subsection{Detection Performance}

Looking at the detection and false alarm probabilities, we can now evaluate the detection performance of the distributed sequential spectrum sensing. Suppose the $n^{\text {th }}$ CR node made a decision $d_{n}$ given by [30]: 


$$
d_{n}= \begin{cases}1 & \text { if } \Lambda\left({ }_{y_{n}}, d_{n-1}\right) \geq \lambda \\ 0 & \text { if } \Lambda\left({ }_{y_{n}}, d_{n-1}\right)<\lambda\end{cases}
$$

Depending on $d_{n-1}, \mu$ can be $\mu_{1}$ or $\mu_{2}$, then the detection and false alarm probabilities are expressed as:

$$
\begin{aligned}
& P_{d, n}=\mathrm{P}_{r}\left\{d_{n}=1 \mid H_{1}\right\}\left(1-P_{d, n-1}\right)+\mathrm{P}_{r}\left\{d_{n}=1 \mid H_{1}\right\} P_{d, n-1} \\
& P_{f a, n}=P_{r}\left\{d_{n}=1 \mid H_{0}\right\}\left(1-P_{f a, n-1}\right)+P_{r}\left\{d_{n}=1 \mid H_{0}\right\} P_{f a, n-1}
\end{aligned}
$$

Since the observations represent random variables, from statistical decision theory, Likelihood ratio test (LRT) is known to be optimal in this type of detection problem [34]. The LRT is implemented using Neyman-Pearson Criterion (maximizing probability of detection but at constraint of false alarm probability)

\subsection{Neyman-Pearson Criterion}

A complete statistical description or analysis of the received signal and noise is required by the Neyman-Pearson detector to predict the presence or absence of a primary signal [35]. The detector may not have a priori information of the received signal and even if available the signal may be varying with time (not stationary).

The Neyman-Pearson Criterion [35] demands that $P_{f a}$ kept fixed to a certain value $\alpha$ and $P_{d}$ is maximized. Since the probability of miss detection $P_{m d}=1-P_{d}$, minimizing $P_{m d}$ is equal to maximizing $P_{d} . \quad P_{d}$ is maximized subject to $P_{f a}=\alpha$ constraint. The decision rule is [26]:

$$
\Lambda(y)=\frac{f_{\mathrm{Y} \mid H_{1}}\left(y \mid H_{1}\right)}{f_{\mathrm{Y} \mid H_{0}}\left(y \mid H_{0}\right)} \underset{H_{0}}{\stackrel{H_{1}}{<}} \lambda
$$

As the threshold $\lambda$ is varied, the regions representing $P_{d}$ and $P_{f a}$ also varies. For a given probability of false alarm, the probability of detection increases. Since $\Lambda(y)$ is a random variable, and the Neyman-Pearson's receiver use the likelihood ratio test, then $P_{d}$ and $P_{f a}$ may be expressed as:

$$
P_{d}\left(\text { Decide } H_{1} \mid H_{1} \text { true }\right)=\int_{\lambda}^{\infty} f_{\Lambda \mid H_{1}}\left(\lambda \mid H_{1}\right) d \lambda
$$

And

$$
P_{f a}\left(\text { Decide } H_{1} \mid H_{0} \text { true }\right)=\int_{\lambda}^{\infty} f_{\Lambda \mid H_{0}}\left(\lambda \mid H_{0}\right) d \lambda
$$

\subsection{Threshold Detection}

The received signal is compared to a threshold value and the detector decides the presence of a primary signal when the signal exceeds the threshold, otherwise it declares the absence of a primary signal. $\Lambda(y)$ represent a ratio of two negative quantities, $f_{Y \mid H_{1}}\left(y \mid H_{1}\right)$ and $f_{Y \mid H_{0}}\left(y \mid H_{0}\right)$, hence takes a value from zero to infinity. When the threshold is zero $(\lambda=0), H_{1}$ hypothesis is true, and $P_{d}=P_{f a}=1$. Similarly, when the threshold tends to infinity $(\lambda \rightarrow \infty)$, $H_{0}$ hypothesis is true, and $P_{d}=P_{f a}=0$ [26]. 
The receiver operating characteristic $(\mathrm{ROC})$ slope at a specific location on the curve specifies the threshold in order to attain $P_{d}$ and $P_{f a}$ at that location or point using the NeymanPearson test [35].

With respect to the threshold $\lambda$ and taking the derivative of equation (14) and (15), we obtain:

$$
\begin{aligned}
& \frac{d P_{d}}{d \lambda}=\frac{d}{d \lambda} \int_{\lambda}^{\infty} f_{\Lambda \mid H_{1}}\left(\lambda \mid H_{1}\right) d \lambda=-f_{\Lambda \mid H_{1}}\left(\lambda \mid H_{1}\right) \\
& \frac{d P_{f a}}{d \lambda}=\frac{d}{d \lambda} \int_{\lambda}^{\infty} f_{\Lambda \mid H_{0}}\left(\lambda \mid H_{0}\right) d \lambda=-f_{\Lambda \mid H_{0}}\left(\lambda \mid H_{0}\right)
\end{aligned}
$$

Also,

$$
P_{d}(\lambda)=P\left[\Lambda(y) \geq \lambda \mid H_{1}\right]=\int_{\lambda}^{\infty} f_{\Lambda \mid H_{1}}\left[\lambda(y) \mid H_{1}\right] d \lambda=\int_{\lambda}^{\infty} \Lambda(y) f_{\Lambda \mid H_{0}}\left[\lambda(y) \mid H_{0}\right] d \lambda
$$

Again with respect to the threshold $\lambda$, a derivative of the above equation is taken to obtain,

$$
\frac{d P_{d}}{d \lambda}=-\lambda f_{\Lambda \mid \mathrm{H}_{1}}\left(\lambda \mid \mathrm{H}_{0}\right)
$$

Combining (16), (17) and (18), yields:

$$
\begin{aligned}
& \frac{f_{\Lambda \mid H_{1}}\left(\lambda \mid H_{1}\right)}{f_{\Lambda \mid H_{0}}\left(\lambda \mid H_{0}\right)}=\lambda \\
& \Rightarrow \quad \frac{d P_{d}}{D P_{f a}}=\lambda
\end{aligned}
$$

\subsection{Nakagami-m Fading Channel}

In a Nakagami fading channel, the probability density function $\gamma$ is given by [30]:

$$
f_{\gamma}(\gamma)=\frac{1}{\Gamma(m)}\left(\frac{m}{\bar{\gamma}}\right)^{m} \gamma^{m-1} \exp \left(-\frac{m \gamma}{\bar{\gamma}}\right) \text { for } \gamma \geq 0
$$

Where $\bar{\gamma}=E[\gamma]$ and $m$ is the Nakagami parameter. Also, the closed-form expression for the probability of detection is given by [8]:

$$
P_{d}=A_{1} \beta^{m} \exp \left(-\frac{\lambda}{2}\right) \sum_{u=1}^{N-1} \frac{(\lambda / 2)^{u}}{u}{ }_{1} F_{1}\left(m ; 1+u ; \frac{\lambda(1-\beta)}{2}\right)
$$

Where $\beta=m /(m+\bar{\gamma})$ and ${ }_{1} F_{1}(. ; ; ;)$ represents the confluent hypergeometric function.

Also, $A_{1}$ for integer values of $m$ is expressed as:

$$
A_{1}=\exp \left(-\frac{\lambda \beta}{2 m}\right)\left[\beta^{m-1} L_{m-1}\left(\frac{-\lambda(1-\beta)}{2}\right)+(1-\beta) \sum_{i=0}^{m-2} \beta^{i} L_{i}\left(\frac{-\lambda(1-\beta)}{2}\right)\right]
$$

Where the Laguerre polynomial of degree $i$ is $L_{i}($.$) .$ 
Also, a closed-form expression for the probability of detection for Nakagami-m fading channel considering Gamma distributed shadowing model is expressed as [36]:

$$
\begin{aligned}
& \overline{P_{d}}=\frac{1}{\Gamma(k)}\left(\frac{m}{\Omega}\right)^{k}\left[\exp \left(-\frac{\lambda}{2}\right) \sum_{s=0}^{\infty} \sum_{n=1}^{u-1} \frac{\left(\frac{\lambda}{2}\right)^{n+s}}{n ! s !} \frac{(m)_{s}}{(n+1)_{s}} \Gamma(s+k) U\left(s+k ; k-m+1 ; \frac{m}{\Omega}\right)\right. \\
& +\sum_{t=0}^{\infty} \sum_{v=0}^{m-2} \frac{(-1)^{t}\left(\frac{\lambda}{2}\right)^{t+v} \Gamma(v+k)}{t ! v !}\left(\begin{array}{c}
m-1 \\
m-v-1
\end{array}\right) U\left(v+k ; k-t-m+2 ; \frac{m}{\Omega}\right) \\
& -\sum_{s=0}^{\infty} \sum_{n=0}^{m-2} \sum_{a=0}^{n} \frac{(-1)^{s}\left(\frac{\lambda}{2}\right)^{a+s}}{a ! s !}\left(\begin{array}{c}
n \\
n-a
\end{array}\right) \Gamma(a+k+1) U\left(a+k+1 ; k-s-n+1 ; \frac{m}{\Omega}\right)
\end{aligned}
$$

For the special case of $m=1$ the Nakagami fading tends to Rayleigh fading. For $m>1$, the signal fluctuations reduces compared to Rayleigh fading and Nakagami fading tends to Rician fading. The Nakagami-m distribution model is a general model which is used widely to describe several fading environments [37].

\section{Results and Analysis}

In this section, simulation results are presented to evaluate the proposed model. The fading and shadowing effects are considered in the simulation. The cooperative nodes are assumed to be distributed sequentially according to the system model.

In order to plot the ROC curves, one parameter is varied while the other parameter is fixed. This allows the assessment of different scenario of interest. The simulation parameters employed are: number of CR users, number of collected samples (n), number of Monte Carlo events $=10,000$, variable SNR and decision threshold. Since the Nakagami fading includes both Raleigh and Rician fading, the Nakagami channel model has been selected for synthesizing the channel (Nakagami fading =1). The threshold is obtained according to equation (21).

As shown in Figure 2, there is an improved detection performance with increasing number of collected samples and increasing number of CR users (cooperative nodes).

The same parameters are also used to assess the effect of $P_{d}$ and $P_{f a}$ against threshold over Nakagami-m fading as shown in Figure 3. As evident, for a higher maximum threshold, the smaller the value of $P_{d}$ and $P_{f a}$. Similarly, the smaller the minimum threshold, $P_{d}$ and $P_{f a}$ increases. Thus, the threshold is treated as an optimization problem, for this study the threshold was set at 0.8 based on the Neman Pearson criterion.

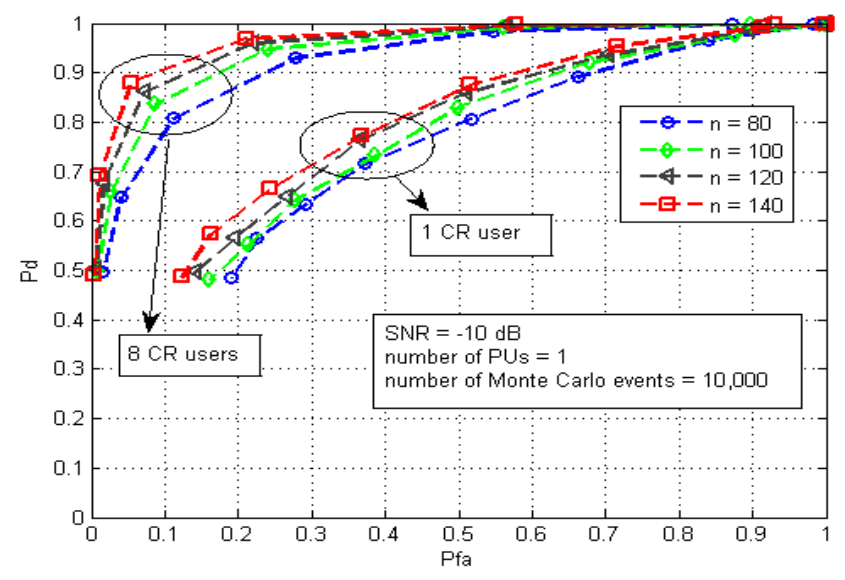

Figure 2. $P_{d}$ vs $P_{f a}$ curves over Nakagami-m fading for variable number of samples (n) and variable number of $\mathrm{CR}$ users (cooperative nodes) 


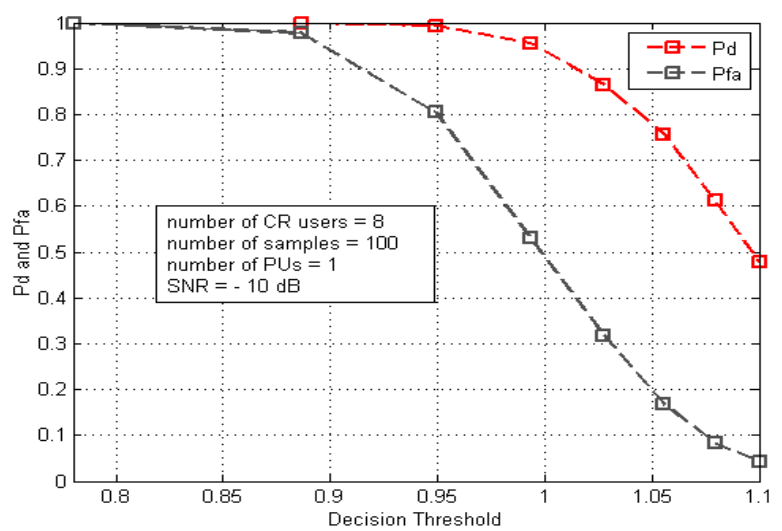

Figure 3. $P_{d}$ and $P_{f a}$ versus variable decision threshold

Finally, the same scenario is used to assess the probability distribution of the system as a function of SNR by showing the probability density function for a fixed threshold value versus variable threshold. Figure 4 and 5 shows the PDF of the test statistic or likelihood ratio obtained from the simulation model using energy detection. The histogram depicts hypothesis.

$H_{0}$ (Primary user absent) and hypothesis $H_{1}$ (primary user present). One can observe that the detection performance of Figure 4 is very low considering low SNR $(-10 \mathrm{~dB})$. The same situation is used for Figure 5 but the SNR is increased to $(0 \mathrm{~dB})$, one can easily see that the $P_{d}$ increases with increase in SNR value, considering that the area representing the histogram under $H_{1}$ increases. The overall conclusion is that for a given reduced $P_{f a}$, there is an increased or improved $P_{d}$. Thus consistent with results in literature.

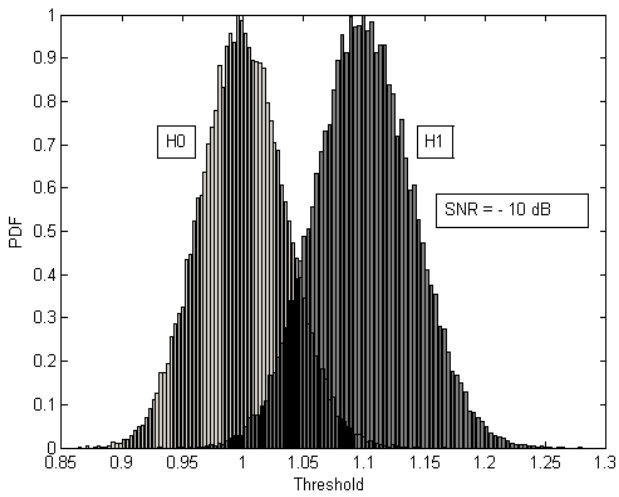

Figure 4. Probability density functions of the test statistic versus variable threshold

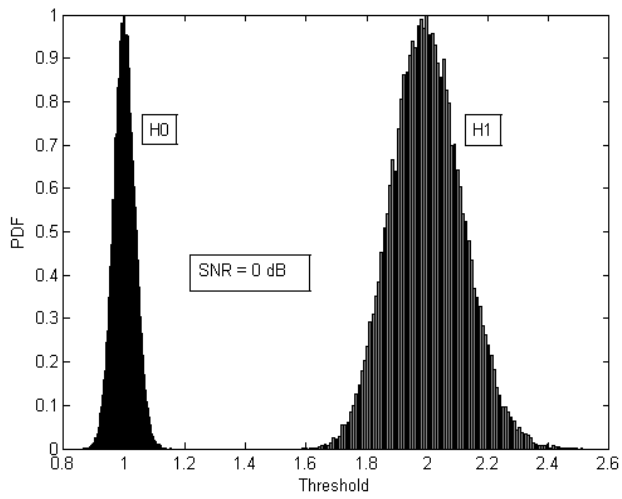

Figure 5. Probability density functions of the test statistic versus variable threshold

\section{Conclusion}

This research paper has studied and proposed a detection technique capable of solving the hidden node problem. The study also reviewed extensively statistical decision theory in relation to spectrum sensing for cognitive radio networks. The derivation of energy detection performance for different types of fading channels have been revisited. We derived closed-form expressions for the probability of detection, the probability of false alarm, over Nakagami-m fading considering Gamma distributed shadowing model for the proposed system. The performance of the proposed method is evaluated by MATLAB simulations to assess the 
influence of cooperation among CR nodes and system parameters such as SNR, decision threshold and number of samples per CR node. The idea is to validate the numerical analysis with simulation results. Simulation results show an improved detection performance with increasing number of samples per CR node and increased number of CR users. A closer observation of the result shows that the probability of detection increases with increased SNR and an increased detection performance for a given reduced false alarm probability. The result also reveals that for a higher maximum threshold, the smaller the value of $P_{d}$ and $P_{f a}$.

Similarly, the smaller the minimum threshold, the higher the value of $P_{d}$ and $P_{f a}$. By implementing energy detector-based distributed sequential cooperative sensing, over Nakagami-m fading, the hidden node problem in identifying the primary user will be greatly reduced. More robust models and channel conditions can be investigated for future works.

\section{References}

[1] J Lunden, V Koivunen, HV Poor. Spectrum exploration and exploitation for cognitive radio: Recent advances. IEEE Signal Processing Magazine. 2015; 32: 123-140.

[2] AK Mishra, DL Johnson. White Space Communication: Advances, Developments and Engineering Challenges. Springer. 2014.

[3] IF Akyildiz, WY Lee, MC Vuran, S Mohanty. NeXt generation/dynamic spectrum access/cognitive radio wireless networks: a survey. Computer Networks. 2006; 50: 2127-2159.

[4] HV Kumar, M Giriprsad. A Novel Approach to Optimize Cognitive Radio Network Utilization using Cascading Technique. TELKOMNIKA Indonesian Journal of Electrical Engineering. 2015; 13: 12331241.

[5] R Umar, AU Sheikh. Spectrum access and sharing for cognitive radio. Developments in wireless network prototyping, design and deployment: Future generations. 2012: 241-271.

[6] R Umar, AU Sheikh. A comparative study of spectrum awareness techniques for cognitive radio oriented wireless networks. Physical Communication. 2013; 9: 148-170.

[7] AA Tabassam, MU Suleman, S Kalsait, S Khan. Building cognitive radios in MATLAB Simulink-A step towards future wireless technology. In Wireless Advanced (WiAd). 2011: 15-20.

[8] FF Digham, MS Alouini, MK Simon. On the energy detection of unknown signals over fading channels. IEEE Transactions on Communications. 2007; 55: 21-24.

[9] N Yadav, S Rathi. A comprehensive study of spectrum sensing techniques in cognitive radio. International Journal of Advances in Engineering \& Technology. 2011: 2231-1963.

[10] A Fanan, N Riley, M Mehdawi, M Ammar, M Zolfaghari. Survey: A Comparison of Spectrum Sensing Techniques in Cognitive Radio.

[11] T Yucek, H Arslan. A survey of spectrum sensing algorithms for cognitive radio applications. Communications Surveys \& Tutorials, IEEE. 2009; 11: 116-130.

[12] S Ziafat, W Ejaz, H Jamal. Spectrum sensing techniques for cognitive radio networks: Performance analysis. In Intelligent Radio for Future Personal Terminals (IMWS-IRFPT), 2011 IEEE MTT-S International Microwave Workshop Series on. 2011: 1-4.

[13] K Aulakh. Spectrum Sensing for Wireless Communication Networks. IEEE. 2009.

[14] K Letaief, W Zhang. Cooperative communications for cognitive radio networks. Proceedings of the IEEE. 2009; 97: 878-893.

[15] R Viswanathan, PK Varshney. Distributed detection with multiple sensors I. Fundamentals. Proceedings of the IEEE. 1997; 85: 54-63.

[16] S Kandeepan, A Giorgetti, M Chiani, Distributed'ring-around'sequential spectrum sensing for cognitive radio networks. In Communications (ICC), 2011 IEEE International Conference on. 2011: 16.

[17] R Viswanathan, S Thomopoulos, R Tumuluri. Optimal serial distributed decision fusion. Aerospace and Electronic Systems, IEEE Transactions on. 1998; 24: 366-376.

[18] JD Papastavrou, M Athans. On optimal distributed decision architectures in a hypothesis testing environment. Automatic Control, IEEE Transactions on. 1992; 37: 1154-1169.

[19] W Huang, X. Xie, Y. Zhang, and S. Zhang, "Research on Sensor Network Spectrum Detection Technology based on Cognitive Radio Network," TELKOMNIKA (Telecommunication Computing Electronics and Control), vol. 14, pp. 101-109, 2016.

[20] K Koufos, K Ruttik, R Jäntti. Distributed sensing in multiband cognitive networks. Wireless Communications, IEEE Transactions on. 2011; 10: 1667-1677.

[21] S Kar, R Tandon, HV Poor, S Cui. Distributed detection in noisy sensor networks. In Information Theory Proceedings (ISIT), 2011 IEEE International Symposium on. 2011: 2856-2860. 
[22] $Y$ Chen, Q. Zhao, and A. Swami, "Distributed spectrum sensing and access in cognitive radio networks with energy constraint," Signal Processing, IEEE Transactions on, vol. 57, pp. 783-797, 2009.

[23] R Chen, JM Park, YT Hou, JH Reed. Toward secure distributed spectrum sensing in cognitive radio networks. Communications Magazine, IEEE. 2008; 46: 50-55.

[24] M Gandetto, C Regazzoni. Spectrum sensing: A distributed approach for cognitive terminals. Selected Areas in Communications, IEEE Journal on. 2007; 25: 546-557.

[25] M Nakagami. The m-distribution-A general formula of intensity distribution of rapid fading. Statistical Method of Radio Propagation. 1960.

[26] MK Simon, MS Alouini. Digital communication over fading channels. John Wiley \& Sons. 2005.

[27] HA Suraweera, GK Karagiannidis. Closed-form error analysis of the non-identical Nakagami-m relay fading channel. IEEE Communications Letters. 2008; 12: 259-261.

[28] C Zhong, T Ratnarajah, KK Wong. Outage analysis of decode-and-forward cognitive dual-hop systems with the interference constraint in Nakagami-fading channels. IEEE Transactions on Vehicular Technology. 2011; 60: 2875-2879.

[29] Y Deng, L Wang, M Elkashlan, KJ Kim, TQ Duong. Generalized Selection Combining for Cognitive Relay Networks Over Nakagami-Fading. IEEE Transactions on Signal Processing. 2016; 63: 19932006.

[30] K Sithamparanathan, A Giorgetti. Cognitive radio techniques: spectrum sensing, interference mitigation, and localization. Artech house. 2012.

[31] SA Kassam. Signal detection in non-Gaussian noise. Springer Science \& Business Media. 2012.

[32] HV Poor. An introduction to signal detection and estimation. Springer Science \& Business Media. 2013.

[33] RR Tenney, NR Sandell. Detection with distributed sensors. IEEE Transactions on Aerospace and Electronic systems. 1981: 501-510.

[34] D Bera, SS Pathak, I Chakrabarty, GK Karagiannidis. Another look in the Analysis of Cooperative Spectrum Sensing over Nakagami Fading Channels. 2015.

[35] M Barkat. Signal detection and estimation. Artech house. 2005.

[36] S Atapattu, C Tellambura, H Jiang. Performance of an energy detector over channels with both multipath fading and shadowing. IEEE Transactions on Wireless Communications. 2010; 9: 36623670.

[37] TT Phu, TD Tran, M Voznak. Analysis of Probability of Non-zero Secrecy Capacity for Multi-hop Networks in Presence of Hardware Impairments over Nakagami-m Fading Channels. Radioengineering. 2016; 25. 